\title{
Ingredients for a Quantum Future
}

\author{
In a series of essays, three quantum-technology leaders in the US spell out \\ the requirements for a strong quantum future.
}

1 rfan Siddiqi will never make a commercial quantum computer-and that's fine by him. The University of

California, Berkeley, physicist has made many contributions to the science of quantum computing by studying the behavior of tiny electronic circuits. But what attracts him to the topic isn't the glory of building a machine. Rather, it's a deep scientific question: Does quantum mechanics work for a large number of degrees of freedom, such as the potentially millions of qubits needed for a quantum computation?

Still, Siddiqi recognizes that if the US is to remain a leader in quantum computing and related technologies, then researchers must avoid forming disconnected islands of knowledge.

Instead, they need to share their ideas, not only with each other but also with industry. "If the task at hand is to study quantum 'at scale," he says, "this [goal] is beyond a small academic lab looking at one little facet."

This perspective is one of several that motivated a series of opinion pieces by Siddiqi and two other quantum-technology leaders-Darío Gil, the director of IBM Research, and Joe Broz, the executive director of the Quantum Economic Development

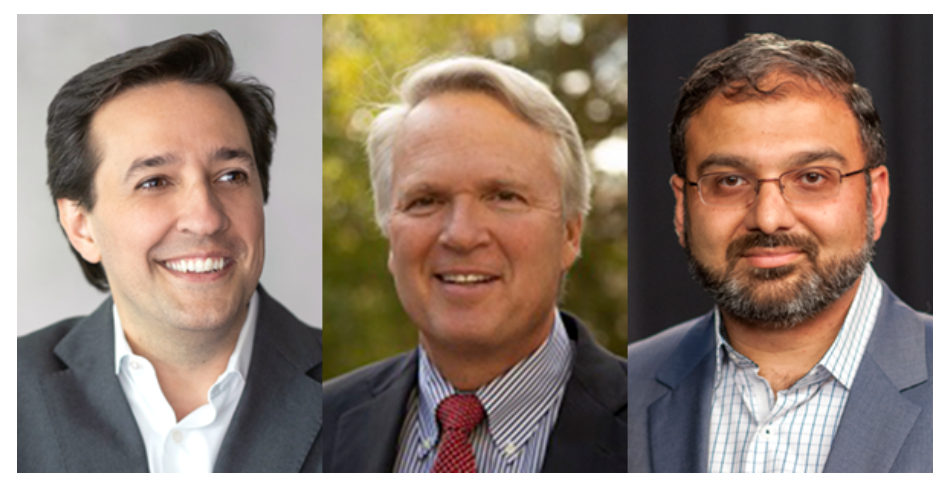

Credit: D. Gil, J. Broz, and I. Siddiqi
Consortium (QED-C). The idea for their articles-the first of which appears today-emerged from conversations between Gil and Siddiqi and, later, Broz, who says that the three of them wanted to clarify "the imperatives that are awaiting the quantum industry as it emerges."

Like China and Europe, the US has invested heavily in quantum computing and other quantum technologies, banking that advances in these areas will transform medicine, encryption, and communication. August 26th saw the announcement of the latest effort, the funding of five Department of Energy (DOE) centers focused on quantum information science. The centers will be run by national labs and each will receive $\$ 125$ million over the next five years.

Siddiqi, Gil, and Broz are enthusiastic about quantum computing, which they view as a transformative technology. "It's not every day that you get a chance to say, hey, something really, really fundamental is happening in the world of information and computing," Gil says. But investment isn't enough. The purpose of the opinion pieces, says Gil, is to "raise the flag" to researchers and industry players that universities, national labs, and industry will need to work together-perhaps in ways they aren't used to. And they will need to be efficient and study the right problems.

The first of the opinion pieces looks at the need for a "culture shift" in how the US approaches quantum computing. The next two essays-one on democratizing quantum knowledge, the other on achieving scalability-will roll out over the next two weeks.

-Jessica Thomas, Editor

See the three essays in this series: A Quantum Culture Shift, Quantum for All, and Quantum at Scale. 
